\title{
Robotic ventral mesh rectopexy for rectal prolapse: a few years until this becomes the gold standard
}

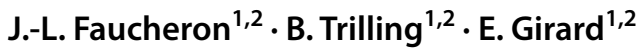

Received: 11 June 2019 / Accepted: 13 June 2019 / Published online: 4 July 2019

(c) Springer Nature Switzerland AG 2019

Several procedures have been described to treat rectal prolapse. The aims of the surgical treatment are to correct the anatomical abnormality, to cure the accompanying symptoms such as incontinence, mucus discharge, bleeding, constipation, and pain, with the lowest rate of complications as possible, an acceptable rate of recurrence, at the lowest possible cost. These goals can be best achieved by ventral rectopexy to the promontory, so that this procedure has become one of the most strongly advocated surgical treatments for patients with full-thickness rectal prolapse and deep enterocele [1]. Surgical treatment of intussusception or isolated rectocele (meaning without enterocele or sigmoidocele on defecography) associated with outlet obstructive defecation is still debated, first depending on which is the primum movens of the symptoms: long-term dyschesia with hard stools leading to intussusception or rectocele should not be an indication for surgery, as "correction" of the anatomy will not cure the functional disorder of obstructed defecation and lead to recurrence due to repeated, prolonged, intense defecatory thrust [2]. Moreover, we know from the literature that some radiologic features, particularly those of internal rectal prolapse (or intussusception) and rectocele, can be present in a third of normal individuals. Specific symptoms have to be present and secondary to the image of "true" prolapse, in order for the condition to be considered pathological. Therefore, some papers from the literature must be interpreted with caution, keeping in mind the proportion of patients with either external rectal prolapse or intussusception, tools that were used to affirm the diagnosis, surgical technique that were used, and postoperative technical and functional results that were observed.

J.-L. Faucheron

JLFaucheron@chu-grenoble.fr

1 Colorectal Unit, Department of Surgery, Michallon University Hospital, CS 10217, 38043 Grenoble cedex, France

2 University Grenoble Alpes, Grenoble, France
With broad availability of surgical robots in major institutions, colorectal applications including rectal prolapse surgery are at the forefront of our minds [3, 4]. Preliminary results of a short series of patients who were operated on for full-thickness rectal prolapse or symptomatic deep enterocele using ventral laparoscopic rectopexy and Douglassectomy with the Da Vinci robot we published in this journal [5] showed that management in a day case surgery setting was effective and safe. This study also demonstrated a significantly longer operative time and total time in the operating theater with the robot and a higher cost with the robot. Results of another prospective study comparing laparoscopic $(n=12)$ versus robot-assisted $(n=16)$ ventral rectopexy in patients presenting with intussusception in the vast majority of cases are published in this issue of the journal [3]. These authors also concluded that robot-assisted ventral mesh rectopexy was more expensive than laparoscopic rectal ventral mesh rectopexy but improvement of health-related quality of life was greater for robotic procedure, that reinforcement of the rectovaginal septum was similar in both techniques, and that the impact of both approaches on long-term generic health-related quality of life was minor, despite symptomatic improvement [3]. The authors attribute the improved results of robotic ventral rectopexy to the more precise fixation of the mesh, despite the fact that the anatomical correction of the posterior vaginal wall was similar. The relative advantage of the robotic approach came with a cost of 39,000 euros per QUALY which is below the usual cutoff point of 46,000 euros. The authors in the discussion make some very thoughtful comments about QALY not being responsive to disease-specific effects of intervention. They also comment on the discrepancy between the significant improvements of the symptom specific quality of life in both groups in the absence of a sustained improvement of the general healthrelated quality of life. Readers should, therefore, consider both positive and negative findings of this trial with caution.

Many authors have compared robot-assisted laparoscopic ventral rectopexy to laparoscopic ventral rectopexy in terms 
of feasibility, safety, cost, and functional results. They did not mention adverse effects due to the use of the Da Vinci robot. Results of robot-assisted laparoscopic rectopexy for rectal prolapse in the long-term, and particularly in elderly patients, reported in the literature did not include any negative impact of the use of the robot. In our experience, however, we could not demonstrate any superiority of robotassisted laparoscopic ventral rectopexy over laparoscopic anterior rectopexy for total rectal prolapse or deep enterocele in terms of technical results and cost [5].

In the year 2019, we could in this editorial make the same remarks concerning robot-assisted ventral rectopexy that we did about robot-assisted total mesorectal excision for cancer [6], commenting on the article from Kim et al. [7].

The first major criticism is patients' selection for the studies about robotic surgery. Following most authors' assertions, one of the advantages of the robot-assisted rectal surgery is mainly the capability for dissection in a narrow and deep pelvis in obese patients. However, most of included patients are women (who have, by anatomical definition, a wide pelvis) with normal body mass index. This is obviously the case in the majority of patients with pelvic floor disorders, who are women with a past history of hysterectomy in nearly one-third of cases [1].

The second major criticism of the articles reporting on robotic rectal surgery is the lack of a frank description of the drawbacks of the robot-assisted rectal surgery. Most of the authors mentioned the uncontrolled assistants' traction as a limitation for the surgical approach to the rectum during the laparoscopic procedures, but they did not comment on the uncontrolled surgeons' traction on tissues because of the total absence of isotonic or isometric force feedback during dissection. In the same way, the use of a "fixed third-arm instrument for retraction" is not always an advantage, once again, because of the absence of force feedback that may lead to bowel tears if the third-arm instrument is out of the screen. The longer time spent for robotic operation should be considered as corresponding to decreased surgical performance and increased physical demands on the surgeon: operative time is always significantly longer in the robotassisted group than in the laparoscopic group. A strong argument for robotic is the three-dimensional ( $3 \mathrm{D}$ ) view of the operative field, but this is only true for the surgeon, not for the assistant. On the contrary, three-dimensional view for the whole team now exists for laparoscopy.

The third major criticism in the literature about robotic rectal surgery is the impossibility to move patient's position when using the robot. Robot-assisted rectal surgery is performed with the patient in the same position throughout the procedure, with no table tilting or rolling as done in open or laparoscopic surgery. Moreover, we need to know in the published series how many patients underwent a hybrid robotic approach, because it is possible that some complications described in the robot-assisted group occurred during laparoscopic phase of the procedure.

The fourth major criticism concerns morbidity. We would like to mention here a deleterious aspect of robotassisted rectal surgery that is rarely published in the literature: If a sudden accident such as significant bleeding should occur, the surgeon is far from the patient and the assistant might be not competent enough to promptly address the problem or even correctly use the suction device to allow prompt hemostasis by the surgeon at the console. We as readers could think that a similar complication could have been more promptly addressed through a laparoscopic approach, with quicker hemostasis induced by the operating surgeon. That is the reason why very often, the first assistant in robotic surgery is a senior and not a junior surgeon. We would like to mention also that for rectal robotic surgery, surgeons use one further port for the assistant, and bigger, metallic ones compared with those used for laparoscopic approach.

In favor of the robotic approach, we would like to argue that most of published series used the first-generation Da Vinci S systems robot. Currently, the third generation of the Da Vinci surgical robot, which is more compact than before, is being developed and used. As this technology evolves, new and more advanced robots will be developed, and undoubtedly will lead to improvements in results of the role of robotic assistance in rectal surgery.

Second, most of the comparative studies were performed at highly specialized laparoscopic centers for colorectal surgery by surgeons with considerable experience in performing laparoscopic rectal surgery with a relatively small number of robotic cases done before beginning the studies. The difference in experience could affect the results and in the future, cases of surgeons with similar experience in laparoscopic and robotic rectal surgeries, robot use could well result in better outcomes.

Third, only randomized, blinded, controlled studies should be performed to provide evidence of the superior role of robots in rectal surgery.

In conclusion, we feel that robot-assisted rectal surgery has several advantages over laparoscopic approach, but most of current published series like the one appearing in this issue have biases that do a disservice to the robotic technique. The fact that some authors declare some conflicts of interests with the robot manufacturer should help readers to interpret with caution the patient's inclusion criteria, the methodology used, and the results published in non-randomized, non-blinded, uncontrolled studies. We are convinced that robot-assisted rectal surgery will probably become the gold standard, but in few years. 


\section{Compliance with ethical standards}

Conflict of interest The author declares that they have no conflict of interest.

Ethical approval This article does not contain any studies with human participants performed by any of the authors.

Informed consent For this type of study, formal consent is not required.

\section{References}

1. Faucheron JL, Trilling B, Girard E, Sage PY, Barbois S, Reche F (2015) Anterior rectopexy for full-thickness rectal prolapse: technical and functional results. World J Gastroenterol 21:5049-5055

2. Trilling B, Faucheron JL (2016) New-onset rectoanal intussusceptions after laparoscopic ventral rectopexy: a normal image? Tech Coloproctol 20:885-886

3. Mäkelä-Kaikkonen J, Rautio T, Ohinmaa A, Koivurova A, Ohtonen P, Sintonen H, Mäkelä J (2019) Cost-analysis and quality of life after laparoscopic and robotic ventral mesh rectopexy for posterior compartment prolapse: a randomized trial. Tech Coloproctol. https://doi.org/10.1007/s10151-019-01991-2

4. Ramage L, Georgiou P, Tekkis P et al (2015) Is robotic ventral mesh rectopexy better than laparoscopy in the treatment of rectal prolapse and obstructed defecation? A meta-analysis. Tech Coloproctol 19:381-389

5. Faucheron JL, Trilling B, Barbois S, Sage PY, Waroquet PA, Reche F (2016) Day case robotic ventral rectopexy compared with day case laparoscopic ventral rectopexy: a prospective study. Tech Coloproctol 20:695-700

6. Faucheron JL, Sage PY, Trilling B (2018) What expert surgeons never tell you about robot-assisted surgery for rectal cancer? Ann Surg. https://doi.org/10.1097/sla.0000000000002986

7. Kim MJ, Park SC, Park JW et al (2018) Robot-assisted versus laparoscopic surgery for rectal cancer: a phase II open label prospective randomized controlled trial. Ann Surg 267:243-251

Publisher's Note Springer Nature remains neutral with regard to jurisdictional claims in published maps and institutional affiliations. 